\title{
Timing Analysis and Optimization for DSM IC-Guest Editorial
}

\author{
CHIEN-IN HENRY CHEN \\ Department of Electrical Engineering, Wright State University, Dayton, OH 45435, USA
}

(Received 15 March 2001; Revised 30 January 2002)

Timing analysis and optimization is one of the key areas in modern VLSI design and has become bottleneck in designing multi-million gate systems. Consequently, computer-aided design research in this area has shown in a new renaissance after the advent of deep sub-micron IC technology and the accompanying restrictive performance constraints. Effects related to interconnect dominance has resulted in technical problems in areas such as floorplanning, placement, routing and performance prediction, and therefore has made accurate and effective timing analysis and optimization more challenging.

The current special issue of VLSI DESIGN Journal is devoted to papers that provide high-quality ideas and results in critical areas related to the timing analysis and optimization for deep sub-micron IC. The first paper called, "Timing Challenges for Very Deep Sub-Micron (VDSM) IC" is by Lin et al. The paper presents current and future timing challenges for VDSM IC. It presents why traditional design flow failed in timing sign-off and how silicon-accurate timing tools are necessary to overcome timing challenges and limitations that cause timing convergence and capacity problems in VDSM IC technology.

The second paper is by Dhaou et al. Its title is "Energy Efficient Signaling in DSM Technology." The key idea is to combat crosstalk noise and to reduce the power consumption while driving the global wire at an optimal delay by reducing voltage swing with buffer insertion and resizing. In $0.25 \mu \mathrm{m}$ CMOS, over $60 \%$ of energy-saving can be achieved if supply voltage is reduced from 2.5 down to $1.5 \mathrm{~V}$.

The third paper is written by Chen et al., entitled "Simultaneous Buffer-Sizing and Wire-Sizing for Clock Tress Based on Lagrangian Relaxation." In this paper, a Lagrangian relaxation algorithm for simultaneously optimizing delay, power, skew, area, and sensitivity in clock tree is presented. The effectiveness and accuracy of algorithm is demonstrated.

The fourth paper is by Hu et al. Its title is "Performance Driven Global Routing Through Gradual Refinement."
The authors present a heuristic for interconnect global routing that can optimize routing congestion, delay and the number of bends that are oftenly competing objectives. In addition to exploiting timing constrained routing flexibility, a gradual refinement method is applied for simultaneous optimization on congestion, timing and the number of bends. Experiments on benchmarks confirm the effectiveness of this method.

The fifth paper is written by Arunachalam et al and is entitled "Accurate Coupling-Centric Timing Analysis Incorporating Temporal and Functional Isolation." This paper presents a timing analysis methodology TACO that can accurately bind the arrival times in the presence of coupling. The timing windows at the aggressor can be used to determine whether the aggressor can switch in conjunction with the victim. Results of industrial examples show that the proposed method actually helps in reducing pessimism in the timing analysis.

The sixth paper is authored by Lee et al. and is entitled "CMOS Delay and Power Model Equations for Simultaneous Transistor and Interconnect Wire Analysis and Optimization." Generalized delay and power equations are proposed in this paper for CMOS circuit optimization achieved by transistor and interconnect minimization. The equations help analyze the entire power-delay trade-off with less complexity and fast computation time.

The seventh paper is written by Huang and is entitled "Improving the Timing of Extended Finite State Machines via Catalyst." In this paper, a timing optimization technique for a complex finite state machine that consists of random logic and data operator is presented. The proposed technique based on the concept of catalyst adds a functionally redundant block to the original circuit so the timing critical paths are divided for speed improvement.

The eighth paper is by Huang et al. Its title is "TimingDriven-Testable Convergent Tree Adders." The tree structure of adders is normally unbalanced and therefore generates high fanout in timing critical paths that may cause racing and increase the delay. A timing optimization 
is presented by reducing the fanout by balancing the tree structure. A full testability of the balanced tree is maintained in the timing optimized tree adder design. A 56-b testable convergent tree adder is presented in this paper.

The ninth paper is written by Xiao et al. and is entitled "Using Temporal and Functional Information in Crosstalk Aware Static Timing Analysis." In this paper, the problem of incorporating temporal and functional information of coupling signals to improve the accuracy of traditional static timing analysis is studied. The technique can be applied to timing driven layout synthesis and optimization. Experimental results demonstrate that the technique significantly reduces the passimism in predicting circuit performance.

The last paper is written by Sulistyo et al. Its title is "A New Characterization Method for Delay and Power Dissipation of Standard Library Cells." A simplified method for characterization of standard cell library cells based on the linear delay model is presented in this paper. The linear model is chosen as it allows rapid characterization with a modest number of simulations, while achieving acceptable accuracy. SPICE simulations on benchmarks show that the timing estimations obtained through the proposed method is accurate to within $5.6 \%$, and the power estimation is accurate to $4.2 \%$.

Its my hope that this special issue will be one of your key references for work in timing analysis and optimization for deep sub-micron IC. Please enjoy reading the special issue!

Chien-In Henry Chen received the B.S. degree from the National Taiwan University, the M.S. degree from the University of Iowa, and the Ph.D. degree from the University of Minnesota, all in electrical engineering. He is currently a Professor of Electrical Engineering at Wright State University at Dayton, OH. From June 1999 to August 2000 he was on leave with Baynacre, Inc., developing silicon-accurate timing verification technologies. His work is primarily in the areas of digital and mixed-signal design synthesis and testing, timing analysis and optimization for VDSM IC, and IC chip design for signal processing, communication and networking. He has written over 70 publications in professional journal and conference proceedings. $\mathrm{He}$ is a technical committee member of the 1995, 1996, 2000-2002 IEEE International ASIC/SOC Conference. He was a plenary speaker of 1995 the 6th VLSI Design/CAD Symposium. 

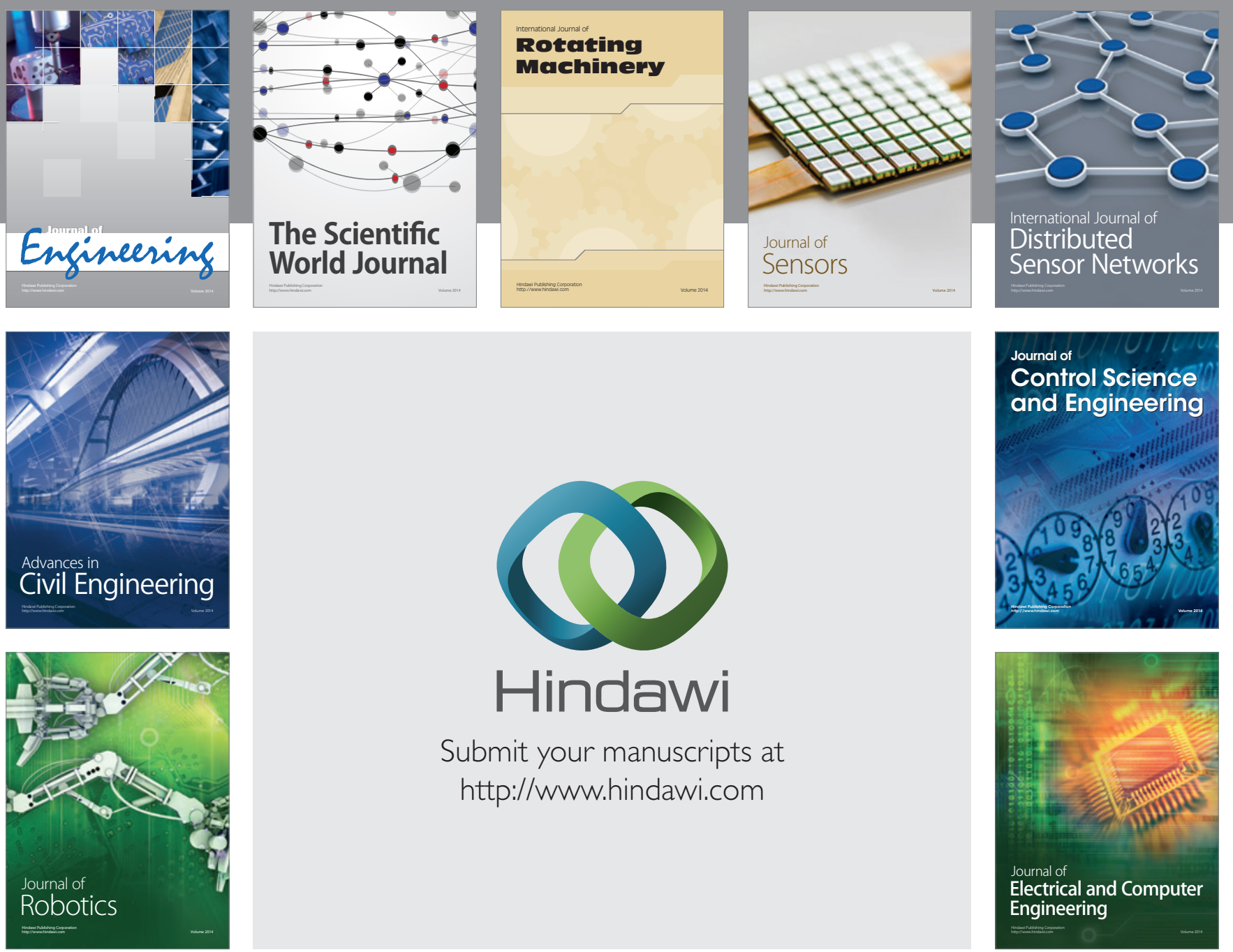

Submit your manuscripts at

http://www.hindawi.com
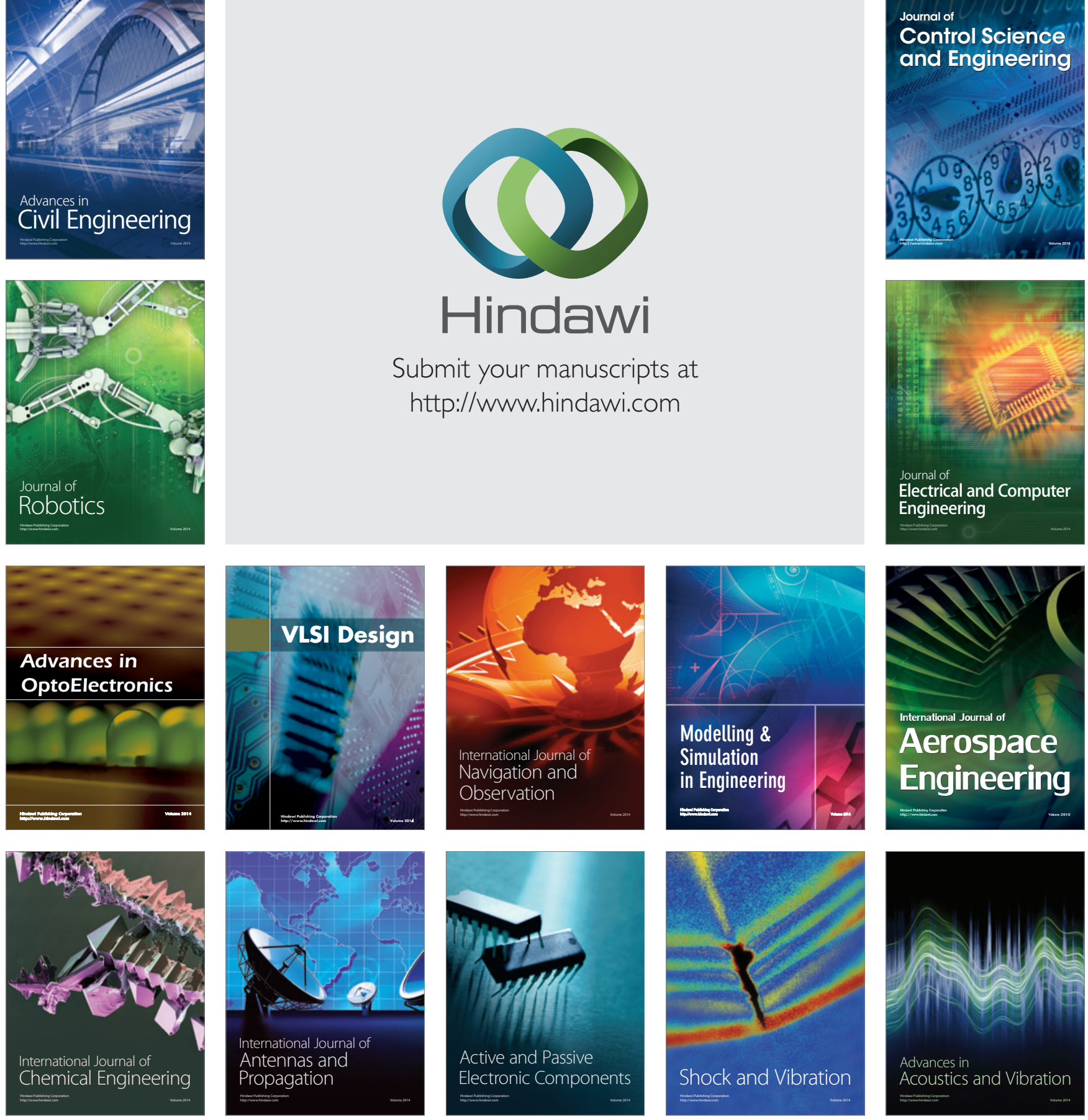